\title{
MULTIDIMENSIONAL INVERSE DIFFERENTIAL QUADRATURE METHOD FOR ANALYSIS OF COMPOSITE PLATE STRUCTURES
}

\author{
$\underline{\text { Hasan M. Khalid }}^{1 *}$, Saheed O. Ojo ${ }^{1}$ and Paul M. Weaver ${ }^{1}$ \\ ${ }^{1}$ Bernal Institute, School of Engineering, University of Limerick, V94 T9PX, Castletroy, Ireland \\ *hasan.m.khalid@ul.ie
}

Mechanics of composite plates are characterised by systems of high-order partial differential equations subject to admissible boundary conditions . Typically, closed form or exact solutions of such high-order systems are rarely feasible or sometimes impossible, thus necessitating a numerical approach. Conventional numerical approaches based on direct approximation of system variables may incur considerable errors subject to high-order numerical differentiation [1]. The inverse differential quadrature method (iDQM) is a new promising approach for obtaining numerical solution of high-order systems based on the approximation of higher order derivatives of a function as linear weighted sum of the function derivatives over the discretized domain [2]. To obtain the iDQM coefficients, a novel routine based on the inversion of existing DQM formula is adopted leading to an efficient and numerically stable scheme (known as $i D Q M$ by-inversion ) which retains the accuracies of high-order secondary variables like strains, and consequently stresses, that are prone to numerical error due to differentiation operations [2]. In this study, two-dimensional iDQM scheme is implemented for static analysis of specially orthotropic composite plate, with inplane dimensions $a$ and $b$, subjected to uniformly distributed transverse load and simply supported boundary condition on all the edges, based on classical laminated plate theory (CLPT). Stress estimates, shown in figure 1, by iDQM of different orders and DQM, benchmarked against closed-form Navier solutions show good agreement with faster convergence.
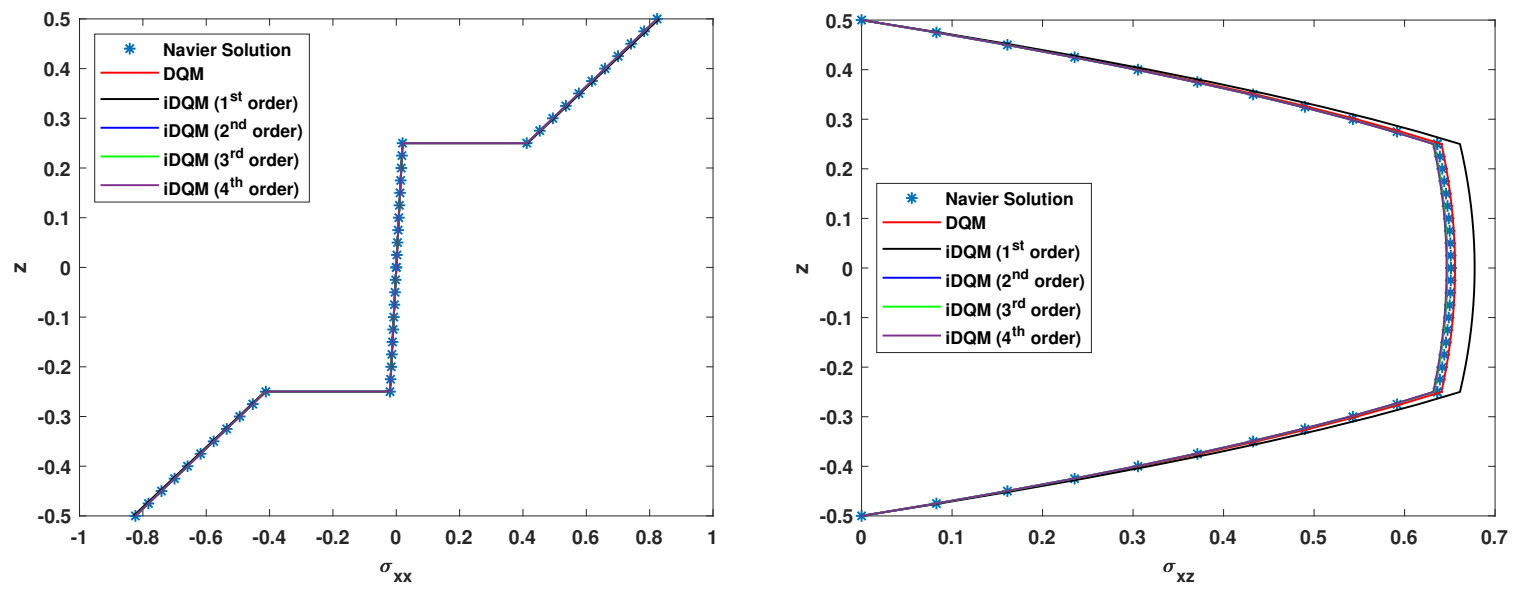

Figure 1: Nondimensionalised $\sigma_{x x}(a / 2, b / 2)$ and $\tau_{x z}(0, b / 2)$ through-the-thickness profiles.

\section{References}

[1] N. Mai-Duy and T. Tran-Cong (2003) Approximation of function and its derivatives using radial basis function networks. Applied Mathematical Modelling, Vol. 27, No. 3 , 197-220,

[2] S. O. Ojo, L. C. Trinh, H. M. Khalid, P. M. Weaver. "Inverse differential quadrature method: mathematical formulation and error analysis". Submitted to Proceedings of Royal Society A (Under review). 